\title{
Orientational dynamics of fluctuating dipolar particles assembled in a mesoscopic colloidal ribbon
}

\author{
Helena Massana-Cid, ${ }^{1}$ Fernando Martinez-Pedrero, ${ }^{2}$ Andrejs Cebers, ${ }^{3}$ and Pietro Tierno ${ }^{1,4,5, *}$ \\ ${ }^{1}$ Departament de Física de la Matèria Condensada, Universitat de Barcelona, 08028 Barcelona, Spain \\ ${ }^{2}$ Departamento de Química Física I, Universidad Complutense de Madrid, Ciudad Universitaria, 28040, Madrid, Spain \\ ${ }^{3}$ Faculty of Physics and Mathematics, University of Latvia, Zellu 23, LV-1002 \\ ${ }^{4}$ Universitat de Barcelona Institute of Complex Systems (UBICS), Universitat de Barcelona, 08028 Barcelona, Spain \\ ${ }^{5}$ Institut de Nanociència i Nanotecnologia, IN ${ }^{2}$ UB, Universitat de Barcelona, 08028 Barcelona, Spain
}

(Received 13 June 2017; published 24 July 2017)

\begin{abstract}
We combine experiments and theory to investigate the dynamics and orientational fluctuations of ferromagnetic microellipsoids that form a ribbonlike structure due to attractive dipolar forces. When assembled in the ribbon, the ellipsoids display orientational thermal fluctuations with an amplitude that can be controlled via application of an in-plane magnetic field. We use video microscopy to investigate the orientational dynamics in real time and space. Theoretical arguments are used to derive an analytical expression that describes how the distribution of the different angular configurations depends on the strength of the applied field. The experimental data are in good agreement with the developed model for all the range of field parameters explored. Understanding the role of fluctuations in chains composed of dipolar particles is important not only from a fundamental point of view, but it may also help understanding the stability of such structures against thermal noise, which is relevant in microfluidics and laboratory-on-a-chip applications.
\end{abstract}

DOI: 10.1103/PhysRevE.96.012607

\section{INTRODUCTION}

Brownian particles assembled into a linear chain due to anisotropic interactions, as the ones arising from dipolar forces, represent an accessible and thus appealing model system to study the role of noise in simple polymerlike structures [1]. When the linkage between the particles is not provided by a strong chemical bond [2-6], but it results from a weak attractive interaction $[7,8]$, then the fluctuations of the single particles may significantly influence the chain dynamics, producing torsions, bending, or even irreversible breakage. Investigating the role of thermal noise in such systems, and how the single-particle fluctuations affect the chain dynamics, is thus necessary to understand the behavior and the stability of the whole structure.

There are different works that explored the deformations and the dynamics of chains composed by spherical microspheres [9-12]. More recently, experiments with magnetic dumbbells [13], Janus rods [14], and hematite ellipsoids [15] have shown the possibility to realize and manipulate elongated structures composed by anisotropic colloids via external fields. In contrast to spherical colloids, anisotropic particles such as ellipsoids introduce an additional rotational degree of freedom that complicates their dynamics, giving rise to a richer physical behavior. However, the role of thermal fluctuations in the orientation of the anisotropic elements when assembled into linear chains has not been addressed yet.

In this article, we study the dynamics of ferromagnetic microellipsoids around the direction determined by an external field, both alone and when assembled into a ribbon. In the latter case, the orientational fluctuations are described by formulating a theoretical framework based on a modified version of the wormlike model [1]. The model allows capturing the fundamental physics of the process and deriving an analytic

\footnotetext{
*ptierno@ub.edu
}

expression for the distribution of the particle orientations within the ribbon.

\section{EXPERIMENTAL SYSTEM AND PROCEDURES}

The anisotropic ferromagnetic ellipsoids are synthesized following a well-established procedure developed by Sugimoto and coworkers [16] and described in detail in several previous works [17-20]. With this method, we obtain monodisperse hematite particles with prolate shape and a major (minor) axis equal to $a=1.80 \pm 0.11 \mu \mathrm{m}(b=1.31 \pm$ $0.12 \mu \mathrm{m}$, respectively). From the analysis of scanning electron microscopy images, we measure a polydispersity index equal to $\sigma_{a}=0.022$ and $\sigma_{b}=0.023$, for the long and short axis of the synthesized particles, respectively. After synthesis, the particles have a small permanent moment $m \simeq 2 \times 10^{-16} \mathrm{Am}^{2}$, perpendicular to their long axis, as depicted in Fig. 1(a). The peculiar orientation of this moment, as compared to other anisotropic magnetic particles [21], is caused by the magnetic structure of hematite, which crystallizes in the corundum form [22]. The value of $m$ was obtained by measuring the reorientational motion of individual hematite particles subjected to a static magnetic field [15]. The permanent moment of the particles allows us to estimate a dipolar coupling constant $\lambda=\mu_{0} m^{2} /\left(4 \pi k_{B} T b^{3}\right)=0.44$ and a Langevin parameter equal to $\Lambda=\mu_{0} m H /\left(k_{B} T\right)=93.2$, for an external field $H=1500 \mathrm{Am}^{-1}$ [23]. Here, $\mu_{0}=4 \pi 10^{-7} \mathrm{Hm}^{-1}$ is the magnetic permeability, $T \sim 293 \mathrm{~K}$ is the room temperature, and $k_{B}$ is the Boltzmann constant. Moreover, from the superconducting quantum interference devices measurements (data not shown here), we find that the particle permanent moment is one order of magnitude larger than the induced moment in all the range of explored field strengths [24].

After synthesis, the particles are dispersed in highly deionized water (purified using a Milli-Q system, Millipore), stabilized with a surfactant by adding $0.11 \mathrm{~g}$ of sodium dodecyl sulfate for $80 \mathrm{ml}$ of water, and finally the $\mathrm{pH}$ of the solution 
(a)
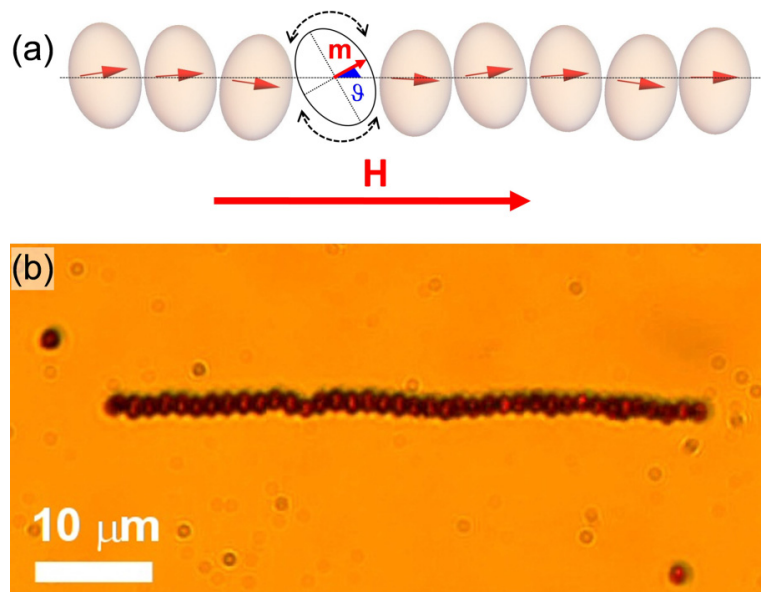

FIG. 1. (a) Schematic showing a chain of ferromagnetic ellipsoids subjected to an external magnetic field $\boldsymbol{H}$, being $\vartheta$ the angle between the particle moment and the $x$ axis, that coincides with the direction of the applied field. Each particle displays orientational thermal fluctuations around this axis. (b) Optical microscope image of a chain composed by 39 ellipsoids under a constant field $H=1600 \mathrm{Am}^{-1}$, applied along the same direction as shown in the schematic at the top.

is adjusted to 9.5 by adding tetramethylammonium hydroxide. These procedures are used to create a protective steric layer around the particles that avoids the irreversible sticking due to attractive Van der Waals interactions. The particles sediment close to a glass plate, where they remain quasi-twodimensionally confined due to the balance between gravity and the electrostatic repulsion with the glass surface. The particle dynamics are visualized using an optical microscope (Eclipse Ni, Nikon), and their positions and orientations are recorded with a CCD camera (Scout scA640-74f, Basler) working at 50 fps. The anisotropic shape of the particles allows for monitoring the instantaneous direction of their permanent moment. The external magnetic field is generated with a custom-made coil system connected to a direct current power supply (EL 302RT, TTi).

\section{SINGLE-PARTICLE DYNAMICS}

Before analyzing the orientational fluctuations in the ribbon, we have first characterized the thermal motion of a single ellipsoid in absence and in presence of an external field. The dynamics of an individual ellipsoid in water has been treated in different works [25-29], and thus here will be only briefly described. In the absence of a magnetic field, the anisotropic shape of the particle causes a nontrivial coupling between its rotational and translational motion. In particular, the particle translational diffusion is anisotropic at short times, $t \sim 0 \mathrm{~s}, t$ being the time interval defined with respect to the initial measured position of the ellipsoid. Thus, the dynamics can be described by two different diffusion coefficients, $D_{\|}$ and $D_{\perp}$, characterizing the translational dynamics parallel and perpendicular to the particle long axis, respectively. This behavior arises from the fact that the ellipsoid has two different friction coefficients, $\gamma_{\|}$and $\gamma_{\perp}$, along the direction parallel and perpendicular to its long axis, respectively. Since $\gamma_{\perp}>\gamma_{\|}$ and $D=k_{B} T / \gamma$, one obtains that $D_{\perp}<D_{\|}$. In contrast, after a long time the diffusion becomes isotropic, and the two diffusion coefficients coincide $D_{\perp}=D_{\|}$. The crossover time between both behaviors is given by the rotational diffusion time, $\tau_{\vartheta}=1 /\left(2 D_{\vartheta}\right), D_{\vartheta}$ being the rotational diffusion coefficient. Thus, for time $t<\tau_{\vartheta}$, one has that $D_{\|} \neq D_{\perp}$, since the rotational motion still needs to become important in the particle dynamics. When $t>\tau_{\vartheta}$, the rotational movement erases the directional memory of the particle, and the two diffusion coefficients approach a common value. To determine the characteristic time $\tau_{\vartheta}$ for our particles, we record the motion of several individual ellipsoids $(i=1 \ldots N)$ and extract the particle positions and orientations, $\left(x_{i}(t), y_{i}(t), \vartheta_{i}(t)\right), \vartheta_{i}(t)$ being the angle between the $x$ axis and the particle minor axis. We then determine the corresponding diffusion coefficients from the mean-square displacement (MSD). For the angular variable, the MSD can be written as

$$
D_{\vartheta}(t)=\frac{1}{2 N t}\left\langle\sum_{n=1}^{N}\left[\vartheta_{i}(t)-\vartheta_{i}(0)\right]^{2}\right\rangle .
$$

From these data, we measure $D_{\vartheta}=0.08 \mathrm{rad}^{2} \mathrm{~s}^{-1}$ that corresponds to $\tau_{\vartheta}=6.2 \mathrm{~s}$. We note that this value is larger than what was found for nonmagnetic ellipsoids $\left(\tau_{\vartheta}=3.1 \mathrm{~s}[26]\right)$, probably due to the different aspect ratio and the higher density of the hematite ellipsoids that could hinder diffusion by forcing the particle to stay closer to the substrate, thus increasing the values of the translational and the rotational friction coefficients. For time $t>\tau_{\vartheta}$, we measure a common diffusion coefficient, $D_{\|}=D_{\perp}=0.074 \mu \mathrm{m}^{2} \mathrm{~s}^{-1}$.

An external magnetic field can be used to impede the rotational motion, separating the diffusion coefficients along the directions parallel and perpendicular to the particle's long axis. In this situation, the particle aligns with its short axis (long axis) parallel (perpendicular) to the field direction. Thus, there is no transition toward the isotropic diffusion, and the hematite ellipsoids present an anisotropic motion with two different diffusion coefficients, even at long times. In particular, for a field amplitude of $H=1500 \mathrm{Am}^{-1}$, we find that the diffusion is totally anisotropic, with $D_{\|}=0.112 \mu \mathrm{m}^{2} \mathrm{~s}^{-1}$ and $D_{\perp}=$ $0.037 \mu \mathrm{m}^{2} \mathrm{~s}^{-1}$. We next analyze, first theoretically and later via experiments, the dynamics of a chain of interacting hematite ellipsoids.

\section{THEORETICAL MODEL}

We consider a chain of permanent dipoles aligned along the direction imposed by a constant field $H$ and subjected to thermal fluctuations that disorder the particle orientations by increasing the angle $\vartheta$, Fig. 1(a). We describe this situation by using a modified version of the stretched wormlike model $[1,30,31]$. In our model the energy of the chain is given by

$$
E=\frac{K_{b}}{2} \int_{0}^{L}\left(\frac{d \vartheta}{d l}\right)^{2} d l-M H \int_{0}^{L} \cos (\vartheta) d l,
$$

where $K_{b}$ is the bending constant that takes into account the dipolar interactions between the ellipsoids and can be written as $K_{b}=m^{2} / 2 b^{2}, b$ being the ellipsoid minor axis. Further, $M$ is the particle magnetization per unit length and $L$ is the length of the chain. Our analysis of the chain thermal fluctuations is based on the relation between the propagator of 
the orientation angles of the dipoles and the chain free energy. The orientational distribution function of the system can be written as

$$
P(\vartheta, l)=\int_{0}^{2 \pi} G\left(\vartheta, l \mid \vartheta^{\prime}, l^{\prime}\right) P\left(\vartheta^{\prime}, l^{\prime}\right) d \vartheta^{\prime}
$$

where the function $G$ in the limit $l-l^{\prime} \rightarrow 0$ is given by the Boltzmann distribution:

$$
G\left(\vartheta, l+\Delta l \mid \vartheta^{\prime}, l\right)=\sqrt{\frac{K_{b}}{2 \pi k_{B} T \Delta l}} e^{\left(-\frac{K_{b}\left(\vartheta-\vartheta^{\prime}\right)^{2}}{2 k_{B} T \Delta l}+\frac{M H \cos (\vartheta) \Delta l}{k_{B} T}\right)} .
$$

In the limit $l \rightarrow l^{\prime}$, we may derive the following differential equation for $P$ [31]:

$$
\frac{\partial P}{\partial l}=\frac{1}{2 l_{p}} \frac{\partial^{2} P}{\partial \vartheta^{2}}+\frac{M H \cos (\vartheta)}{k_{B} T} P=\hat{H} P,
$$

where $l_{p}=K_{b} / k_{B} T$ is the persistence length of the chain. As a result, $G$ may be expressed through the eigenfunctions $\psi_{k}$ and the eigenvalues $\lambda_{k}$ of the operator $\hat{H}$ as follows:

$$
G=\sum_{k} \exp \left[\lambda_{k}\left(l-l^{\prime}\right)\right] \psi_{k}(\vartheta) \psi_{k}\left(\vartheta^{\prime}\right) .
$$

For infinitely long chains, $L \rightarrow \infty\left(l=L ; l^{\prime}=0\right)$, only the largest eigenvalue $\lambda_{1}$ will contribute in Eq. (6), and

$$
G\left(\vartheta, L \mid \vartheta^{\prime}, 0\right)=\exp \left(\lambda_{1} L\right) \psi_{1}(\vartheta) \psi_{1}\left(\vartheta^{\prime}\right)
$$

Since

$$
\begin{aligned}
G\left(\vartheta, L \mid \vartheta^{\prime}, 0\right)= & \left.\int \ldots \int G\left(\vartheta, \vartheta_{n-1} \mid \Delta l\right) \ldots G\left(\vartheta_{1}, \vartheta^{\prime} \mid \Delta l\right)\right) \\
& \times d \vartheta_{1} \ldots d \vartheta_{n-1},
\end{aligned}
$$

we thus obtain

$$
\int G\left(\vartheta, L \mid \vartheta^{\prime}, 0\right) d \vartheta d \vartheta^{\prime}=Z,
$$

where $Z$ is a statistical sum done over all the ellipsoids that form the magnetic chain. Thus, the total free energy can be expressed as

$$
F=-k_{B} T \ln Z=-k_{B} T \lambda_{1} L .
$$

For large values of $L$, the orientation distribution function can be determined by the eigenfunction of $\hat{H}$ corresponding to the largest eigenvalue $\lambda_{1}$ :

$$
P(\vartheta) \sim \psi_{1}(\vartheta)
$$

Now we present the results for the two limiting regimes characterized by the amplitude of the applied field.

\section{LARGE APPLIED FIELDS}

Let us consider a magnetic chain with length $L$ under periodic conditions, $\vartheta(l+L)=\vartheta(l)$. We can write the angle $\vartheta$ as

$$
\vartheta=\sum_{k} \vartheta_{k} \exp (i k l) ; k=\frac{2 \pi n}{L}
$$

For small thermal fluctuations, the energy of the system may be expressed as

$$
E=\frac{L}{2} \sum_{k}\left(K_{b} k^{2}+M H\right)\left|\vartheta_{k}\right|^{2}
$$

According to the Boltzmann principle, we can write the probability density of the amplitude of the fluctuations as

$$
\varrho \approx \exp \left(-L \sum_{k>0}\left(K_{b} k^{2}+M H\right)\left|\vartheta_{k}\right|^{2} / k_{B} T\right),
$$

and the spectral amplitude as

$$
\left\langle\left|\vartheta_{k}\right|^{2}\right\rangle=\frac{k_{B} T}{L\left(K_{b} k^{2}+M H\right)},
$$

and

$$
\left\langle\int_{0}^{L} \cos (\vartheta) d l\right\rangle=L-L \sum_{k>0}\left|\vartheta_{k}\right|^{2} .
$$

The sum in Eq. (16) can be calculated upon direct integration,

$$
\left\langle\int_{0}^{L} \cos (\vartheta) d l\right\rangle=L\left(1-\frac{1}{4} \frac{1}{\sqrt{M H K_{b} /\left(k_{B} T\right)^{2}}}\right) .
$$

At equilibrium, the variation of the chain free energy with the magnetic field may be expressed as

$$
\frac{\partial F}{\partial H}=-M\left\langle\int_{0}^{L} \cos (\vartheta) d l\right\rangle,
$$

where the right member in Eq. (18) is the total magnetic moment of the chain. From Eqs. (10), (17), and (18), we derive the following relationship:

$$
k_{B} T L \frac{\partial \lambda_{1}}{\partial H}=M L\left(1-\frac{1}{2 \sqrt{2} \sqrt{\xi}}\right),
$$

which can be used to test our variational approach, $\xi=$ $M H 2 l_{p} / k_{B} T$ being the dimensionless magnetic energy. By defining the dimensionless variable $\tilde{\lambda}_{1}=\lambda_{1} 2 l_{p}$, we have

$$
\frac{\partial \tilde{\lambda}_{1}}{\partial \xi}=1-\frac{1}{2 \sqrt{2} \sqrt{\xi}}
$$

or

$$
\tilde{\lambda}_{1} \simeq \xi-\frac{1}{\sqrt{2}} \sqrt{\xi}
$$

\section{SMALL APPLIED FIELDS}

In the limit of small applied fields, and according to the perturbation theory, the magnetization is given by

$$
\begin{aligned}
& \left\langle\int_{0}^{L} \cos (\vartheta) d l\right\rangle \\
& \quad=Z^{-1} \int D(\vartheta) \int_{0}^{L} \cos [\vartheta(l)] d l \exp \left(-E / k_{B} T\right) \\
& \cong \frac{M H}{k_{B} T}\left\langle\int_{0}^{L} \cos [\vartheta(l)] d l \int_{0}^{L} \cos \left(\vartheta\left(l^{\prime}\right) d l^{\prime}\right\rangle .\right.
\end{aligned}
$$

From Eq. (5), it follows that in the absence of a magnetic field

$$
\left\langle\cos [\vartheta(l)] \cos \left[\vartheta\left(l^{\prime}\right)\right]\right\rangle=\frac{1}{2} \exp \left[-\left|l-l^{\prime}\right| /\left(2 l_{p}\right)\right] .
$$


Integrating the previous equation, one obtains

$$
\int_{0}^{L} \int_{0}^{L} \frac{1}{2} \exp \left[-\left|l-l^{\prime}\right| /\left(2 l_{p}\right)\right] d l d l^{\prime} \simeq 2 l_{p} L .
$$

From Eqs. (22), (23), and (24), the magnetic moment is given by

$$
M\left\langle\int_{0}^{L} \cos (\vartheta) d l\right\rangle=M \frac{M H}{k_{B} T} 2 l_{p} L
$$

and from Eq. (18),

$$
k_{B} T \frac{\partial \lambda_{1}}{\partial H}=M \frac{M H}{k_{B} T} 2 l_{p} .
$$
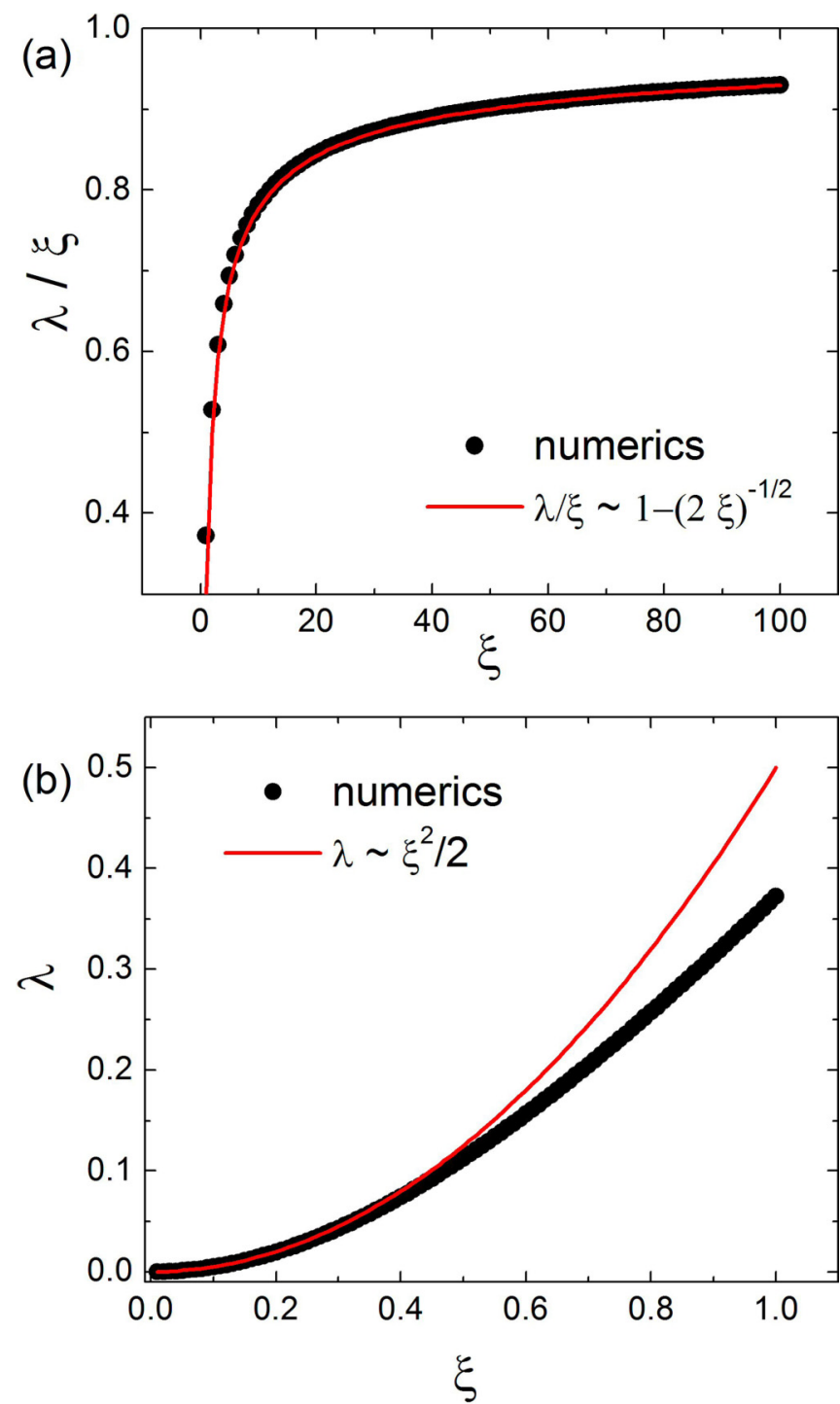

FIG. 2. (a) Ratio $\lambda / \xi$ versus $\xi$, $\lambda$ being the eigenvalues corresponding to the maximum of the functional $W$. The continuous red line is a fit corresponding to Eq. (21), where the numerical data are obtained for large values of $\xi$. (b) Eigenvalues $\lambda$ calculated for small values of $\xi \in[0 \ldots 1]$. The continuous red line corresponds to Eq. (27) in the text.
Finally, we arrive to another relationship that is valid for large thermal fluctuations,

$$
\frac{\partial \tilde{\lambda}_{1}}{\partial \xi}=\xi ; \tilde{\lambda}_{1}=\frac{1}{2} \xi^{2},
$$

and its validity can be demonstrated by using a variational method, as described in the following section.

\section{VARIATIONAL SOLUTION}

By defining the dimensionless length $l / 2 l_{p}$, Eq. (5) follows from the variation of the functional

$$
W=\int_{0}^{2 \pi}\left[-\left(\frac{\partial P}{\partial \vartheta}\right)^{2}+\xi \cos (\vartheta) P^{2}\right] d \vartheta / \int_{0}^{2 \pi} P^{2} d \vartheta .
$$

From this equation, we have

$$
\frac{d^{2} P}{d \vartheta^{2}}+\xi \cos (\vartheta) P=W P,
$$

and the largest eigenvalue of the operator $\hat{H}$ corresponds to the maximal value of the functional $W$. To find this value, we use the following trial function for the probability density distribution:

$$
P=\frac{\exp [\alpha \cos (\vartheta)]}{\sqrt{2 \pi I_{0}(2 \alpha)}},
$$

where $I_{n}(x),(n=0,1,2, \ldots)$ are the modified Bessel functions of the first kind. This function is normalized as $\int_{0}^{2 \pi} P^{2} d \vartheta=1$, and it is proportional to $\exp (\alpha \cos \vartheta)$. Using this expression, the functional in Eq. (28) can be written as

$$
W=\frac{1}{I_{0}(2 \alpha)}\left[-\frac{1}{2} F_{01}\left(2, \alpha^{2}\right)+\xi I_{1}(2 \alpha)\right],
$$

where $F_{01}(2, z)$ is the confluent hypergeometric function. To justify the selection of the trial function, we calculated the values of the parameter $\alpha$ that maximize the functional in Eq. (31) for both small $(\xi \in[0 ; 0.1])$ and large $(\xi \in[10 ; 100])$ values of $\xi$. The data resulting from these calculations are plotted versus $\xi$ in Figs. 2(a) and 2(b). From these images, it follows indeed that the numerical data are in excellent agreement with the theoretical expressions derived from small [Eq. (21)] and large [Eq. (27)] thermal fluctuations.

\section{FLUCTUATIONS IN THE COLLOIDAL RIBBON}

To validate our model, we perform different experiments by measuring the average angle $\vartheta$ as a function of the applied field. Thermally induced torsions, which cause the rotation of the ellipsoids around the main axis of the chain, are hindered by the presence of the glass surface, and almost all the ellipsoids rest on the surface with their long axis parallel to the horizontal plane. In this study, we have also neglected the weak out-of-plane fluctuations of the magnetic moments. Further, we find that during the measurements the magnetic ribbons are sensitive to the Earth's magnetic field, $H_{e} \sim 40 \mathrm{Am}^{-1}$, and thus they orient along $H_{e}$ even in the absence of any extra applied field. To cancel $H_{e}$, we balance the latter by applying a small DC field in the opposite direction. Under this condition, the particles in the chain do not present any preferential orientation, as shown by the empty circles in Fig. 3, 


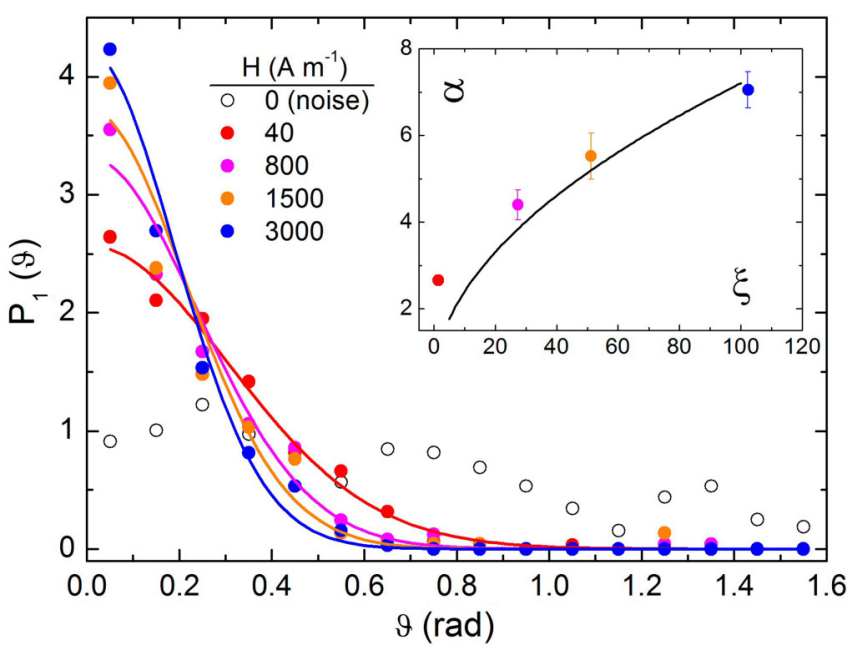

FIG. 3. Distribution of the particle orientation, $P_{1}(\vartheta)$, for different amplitudes of the applied field $H$. Symbols denote experimental data, continuous lines starting from $H=40 \mathrm{Am}^{-1}$ are theoretical curves calculated following the equation of $P_{1}(\vartheta)$ in the text. The obtained values of the fitted dimensionless parameter $\alpha=\tilde{\alpha} / 2$ for all the curves are shown as a function of $\xi=m H l_{p} / b k_{B} T$ in the top inset. Here we use $l_{p}=0.3 \mu \mathrm{m}$ and $m=2.3 \times 10^{-16} \mathrm{Am}^{2}$. The continuous line results from the functional $W$ in Eq. (31).

and the ribbons form rings $[14,20,32]$ or break due to thermal fluctuations.

The distribution of the angles $P_{1}(\vartheta)$ are shown in Fig. 3, where the experimental data are scattered points, while the continuous lines are fit to the theoretical model. Due to the geometry of our experimental system, we have assumed that the distribution is symmetric around the $x$ axis, $P_{1}(\vartheta)=$ $P_{1}(-\vartheta)$, and that $P_{1}(\vartheta>\pi / 2) \sim 0$. Thus, we normalize the experimental data as $\int_{0}^{\pi / 2} P_{1}(\vartheta) d \vartheta=1$ and use the expression $P_{1}(\vartheta)=\exp [\tilde{\alpha} \cos (\vartheta)] /\left[\pi I_{0}(\tilde{\alpha})\right]$. We note that this distribution reduces to Eq. (30) of the model $P_{1}(\vartheta)=$ $P(\vartheta)$ for $\alpha=\tilde{\alpha} / 2$ and large values of the parameter $\alpha$. The application of an external field reduces the amplitude of the orientational fluctuations, centering the angular distribution about $\vartheta=0$ rad. The half width of the distribution decreases with the field, in agreement with the prediction of Eq. (30). The good agreement between the experimental data and the theoretical expression is also proved by the small inset in Fig. 3. Here we show the fitting parameters $\alpha=\tilde{\alpha} / 2$ obtained from the main graph as a function of the normalized magnetic energy $\xi$. To determine $\xi$, we use as persistence length of the chain the value $l_{p} \sim 0.3 \mu \mathrm{m}$, which was previously determined in a set of independent experiments [20]. The scattered data are in excellent agreement with the continuous line that was independently obtained from the numerical calculation of the maximum of the functional $W$ in Eq. (31).

\section{CONCLUSIONS}

We have combined experiments and theory to analyze the orientational fluctuations in a colloidal chain made by ferromagnetic hematite ellipsoids. Our model quantitatively captures the physics of the colloidal system, showing a good agreement with the experimental data. In absence of external field, the chain shows large fluctuations of the individual elements along the whole range of angles $[0,2 \pi]$ and can easily break. When an external field is applied, the amplitude of the fluctuations decreases, becoming limited to a narrow range of angles.

The assembly of magnetic colloids into linear chains is an appealing research subject with both fundamental [33] and technological applications [34]. In the first case, magnetic chains influenced by thermal fluctuations have shown a variety of interesting phenomena, including diffusion limited aggregation [35,36], multiscale kinetics [37], and subdiffusive dynamics [38]. On the application side, magnetic chains have been used in the past as micromechanical sensors [3,39], to measure the growth of actin filaments [40], the rheological properties of the dispersing medium [41,42], or even to realize biomimetic structures, such as actuated magnetic propellers $[43,44]$ and artificial cilia $[45,46]$. Further, our mesoscopic colloidal system may be also used as a simplified model for magnetic polymer beads, which present exciting applications in targeted drug delivery and in the oil industry [47].

\section{ACKNOWLEDGMENTS}

H.M.C., F.M.P., and P.T. acknowledge support from the European Research Council (ERC) starting Grant "DynaMO". P.T. acknowledges support from Ministerio de Economía, Industria y Competitividad (MINECO) (Grant No. FIS201678507-C2-2-P) and Agència de Gestió d'Ajuts Universitaris i de Recerca (DURSI) (Grant No. 2014SGR878). A.C. acknowledges support from National Research Programme Grant No. 2014.10-4/VPP-3/21 and from the M-ERA-NET project "Metrology at the Nanoscale with Diamonds" (MyND). F.M.P. acknowledges support from MINECO (Grant No. RYC-201518495).
[1] M. Doi and S. F. Edwards, The Theory of Polymer Dynamics (Clarendon Press, Oxford, 1988).

[2] E. M. Furst, C. Suzuki, M. Fermigier, and A. P. Gast, Langmuir 14, 7334 (1998).

[3] C. Goubault, P. Jop, M. Fermigier, J. Baudry, E. Bertrand, and J. Bibette, Phys. Rev. Lett. 91, 260802 (2003).

[4] S. L. Biswal and A. P. Gast, Phys. Rev. E 68, 021402 (2003).

[5] H. R. Vutukuri, A. F. Demirörs, B. Peng, P. D. J. van Oostrum, A. Imhof, and A. van Blaaderen, Angew. Chem. Int. Ed. 50, 3747 (2011).
[6] A. Demortière, A. Snezhko, M. V. Sapozhnikov, N. Becker, T. Proslier, and I. S. Aranson, Nat. Commun. 5, 3117 (2014).

[7] A. T. Skjeltorp, Phys. Rev. Lett. 51, 2306 (1983).

[8] R. Toussaint, G. Helgesen, and E. G. Flekkøy, Phys. Rev. Lett. 93, 108304 (2004).

[9] A. S. Silva, R. Bond, F. Plouraboué, and D. Wirtz, Phys. Rev. E 54, 5502 (1996).

[10] S. Cutillas and J. Liu, Phys. Rev. E 64, 011506 (2001).

[11] L. Hong, S. M. Anthony, and S. Granick, Langmuir 22, 7128 (2006). 
[12] D. Li, N. Fakhri, M. Pasquali, and S. L. Biswal, Phys. Rev. Lett 106, 188302 (2011).

[13] D. Zerrouki, J. Baudry, D. Pine, P. Chaikin, and J. Bibette, Nature 455, 380 (2008).

[14] J. Yan, K. Chaudhary, S. C. Bae, J. A. Lewis, and S. Granick, Nat. Commun. 4, 1516 (2013).

[15] F. Martinez-Pedrero, A. Cebers, and P. Tierno, Soft Matter 12, 3688 (2016).

[16] T. Sugimoto, M. M. Khan, and A. Muramatsu, Colloids Surfaces A-Physicochem. Eng. Aspects 70, 167 (1993).

[17] S. J. Gerbode, S. H. Lee, C. M. Liddell, and I. Cohen, Phys. Rev. Lett. 101, 058302 (2008).

[18] S. H. Lee and C. M. Liddell, Small 5, 1957 (2009).

[19] J. Palacci, S. Sacanna, A. Vatchinsky, P. M. Chaikin, and D. J. Pine, J. Am. Chem. Soc. 135, 15978 (2013).

[20] F. Martinez-Pedrero and P. Tierno, Phys. Rev. Appl. 3, 051003 (2015).

[21] P. Tierno, Phys. Chem. Chem. Phys. 16, 23515 (2014).

[22] C. G. Shull, W. A. Strauser, and E. O. Wollan, Phys. Rev. 83, 333 (1951).

[23] R. E. Rosensweig, Ferrohydrodynamics (Cambridge University Press, Cambridge, 1986).

[24] F. Martinez-Pedrero, H. Massana-Cid, and P. Tierno, Small 13, 1603449 (2017).

[25] F. Perrin, J. Phys. Radium 5, 497 (1934).

[26] Y. Han, A. M. Alsayed, M. Nobili, J. Zhang, T. C. Lubensky, and A. G. Yodh, Science 314, 626 (2006).

[27] R. Grima and S. N. Yaliraki, J. Chem. Phys. 127, 084511 (2007).

[28] T. P. Güell O. and S. F., Eur. Phys. J. ST, 187, 15 (2010).

[29] W.-T. Louis Fan, O. S. Pak, and M. Sandoval, Phys. Rev. E 95, 032605 (2017).

[30] M. Fixman and J. Kovac, J. Chem. Phys. 58, 1564 (1973).

[31] M. J. F. and E. D. Siggia, Macromolecules 28, 8759 (1995).

[32] W. Wen, F. Kun, K. F. Pál, D. W. Zheng, and K. N. Tu, Phys. Rev. E 59, R4758(R) (1999).

[33] K. Butter, P. H. H. Bomans, P. M. Frederik, G. J. Vroege, and A. P. Philipse, Nat. Mater. 2, 88 (2006).
[34] B. Berkovski and V. B. (eds.), Magnetic Fluids and Applications Handbook (Begel House, New York, 1996).

[35] J. Černák, G. Helgesen, and A. T. Skjeltorp, Phys. Rev. E 70, 031504 (2004).

[36] R. M. Erb, M. D. Krebs, E. Alsberg, B. Samanta, V. M. Rotello, and B. B. Yellen, Phys. Rev. E 80, 051402 (2009).

[37] J. W. Swan, P. A. Vasquez, P. A. Whitson, E. M. Fincke, K. Wakata, S. H. Magnus, F. D. Winne, M. R. Barratt, J. H. Agui, R. D. Green, N. R. Hall, D. Y. Bohman, C. T. Bunnell, A. P. Gast, and E. M. Furst, Proc. Natl. Acad. Sci. USA 109, 16023 (2012).

[38] J. Jordanovic, S. Jäger, and S. H. L. Klapp, Phys. Rev. Lett. 106, 038301 (2011).

[39] R. Dreyfus, D. Lacoste, J. Bibette, and J. Baudry, Eur. Phys. J. E 28, 113 (2009).

[40] C. Brangbour, O. du Roure, E. Helfer, D. Démoulin, A. Mazurier, M. Fermigier, M.-F. Carlier, J. Bibette, and J. Baudry, PLoS Biol. 9, e1000613 (2011).

[41] G. K. Auernhammer, D. Collin, and P. Martinoty, J. Chem. Phys. 124, 204907 (2006).

[42] S. Huang, G. Pessot, P. Cremer, R. Weeber, C. Holm, J. Nowak, S. Odenbach, A. M. Menzel, and G. K. Auernhammer, Soft Matter 12, 228 (2016).

[43] R. Dreyfus, J. Baudry, M. L. Roper, M. Fermigier, H. A. Stone, and J. Bibette, Nature 437, 862 (2005).

[44] F. Martinez-Pedrero, A. Ortiz-Ambriz, I. Pagonabarraga, and P. Tierno, Phys. Rev. Lett. 115, 138301 (2015).

[45] M. Vilfan, A. Potocnik, B. Kavcic, N. Osterman, I. Poberaj, A. Vilfan, and D. Babic, Proc. Natl. Acad. Sci. USA 107, 1844 (2010).

[46] N. Coq, A. Bricard, F.-D. Delapierre, L. Malaquin, O. du Roure, M. Fermigier, and D. Bartolo, Phys. Rev. Lett. 107, 014501 (2011).

[47] S. E. Kushnir, P. E. Kazin, L. A. Trusov, and Y. D. Tretyakov, Russian Chem. Rev. 81, 560 (2012). 